\title{
Creating Meaningful Grades
}

\author{
Joshua Kunnath \\ Principal Research Center, Inc.
}

Grades matter, and the future lives of students are in many ways dependent on teacher grading practices. After all, so many decisions that affect students' lives, including student ranking, matriculation, retention, college admission, and scholarships, depend on grades (Guskey, 2015; Marzano, 2000). This is troubling because the grading practices used in high schools across the country are generally considered to be highly variable and invalid measures of learning, often consisting of a hodgepodge of factors including achievement, behavior, and effort (Brimi, 2011; Brookhart, 1991; Randall \& Engelhard, 2009, 2010). In other words, the data that many parties use to make important decisions regarding the lives of students is invalid. In fact, Marzano (2000), a leading grading researcher, declared, "Grades are so imprecise that they are almost meaningless" (p. 1). While most parties assume that grades represent student learning (Brookhart, 2004), this is rarely the case. As a result, the misinterpretation of grade data often leads to poor decisions regarding students. To add insult to injury, over 100 years of research has documented these problems (Brookhart et al., 2016), but educators' seeming indifference to the research has resulted in no significant changes (Dueck, 2014; Reeves, 2011).

Doubtlessly some school leaders seek to make positive changes in this area, but they face many challenges to their efforts. Grading is an extremely controversial topic, as teachers traditionally enjoy full autonomy of the practice. As a result, some teachers may feel that any grading reform infringes on a sacred teaching right passed down from Horace Mann himself. Moreover, grading is not a topic that most teachers, students, and parents even see as a problem area. The hodgepodge practices referred to in the literature are all that teachers and parents often know. It is what they experienced as students and it is what they have come to expect in schools teaching their own children. In fact, for many, traditional grading practices are part of what makes school school (Kunnath, 2016; Marzano, 2000). Any reform movement is risky, but attempts to reform practices that are seen in most schools as acceptable, if not ideal, can be especially difficult.

Clearly grading reform is a complex topic, and upon some consideration of both sides of the issue, it seems that a measured approach to reform is most likely to produce desired results. The educator choosing this approach realizes that grading reform is in the best interest of student learning, but that it must also be done tactfully. The reformer seeks to improve the very foundation of grading practices, but she also wants to limit dissension within the school community. For all of these reasons, an opportune beginning to grading reform is the establishment of meaningful grades. By clearly establishing the meaning of student grades, an individual teacher or an entire school can take a big step toward improving the integrity of report card grades and the grading practices that lead up to them.

\section{Why Grades Require an Explicit Meaning}

Without a definitive meaning, grades are nearly worthless. Letter grades have no universal meaning, so for anyone to accurately interpret the feedback that grades are intended to provide, they require teacherdefined meanings. Whether used as scores on assessments or as a summary of a marking period on a report card, grades require a definition that enables the intended audience - primarily students and parents-to clearly understand students' performance in each class. And while it is standard knowledge that an A on a report card represents excellence, one must wonder: What about the student is excellent? Is it achievement? Attendance? Participation? Effort? Behavior? A combination of all of these factors? By establishing a grade meaning, teachers take the guesswork out of grades and add transparency to their practices.

Focusing on grade meaning can also provide teachers with a new lens to view their grading practices, helping them to be more intentional about how they create report card grades. This new way of thinking can then lead to further grading reform efforts such as improving grade accuracy, establishing common 
grading practices, and revising report cards. In this manner, defining grade meaning can be seen as a high -leverage reform. Like any reform, establishing grading meaning is best accomplished when it is aligned to relevant research.

\section{Creating a Research-aligned Grade Meaning}

According to measurement experts, grades should mean one thing: student learning of academic standards (Guskey, 2015; Reeves, 2011). This is, after all, what teachers are best qualified and able to measure. Teachers following this recommendation should create a meaning statement that ensures clarity and transparency of grades. Next, this meaning needs to be communicated to students and parents (Guskey, 2015; Reeves, 2011)--a process that is about actively educating them. The communication process is also about changing grading culture from grades as classroom currency for punishing and rewarding students to grades as data on student learning. The more students and parents buy into this new culture, the more the teacher can focus students' energy on their own learning instead of misguided attention to grade grubbing.

\section{The Grade Meaning Statement}

The grade meaning statement should be concise and straightforward to achieve the purpose of communicating grade meaning to students and parents (Guskey, 2015). Figure 1 displays an example statement appearing on a syllabus in a high school English class. Although the example is simple, it communicates the message that grades equal student learning, and it allows students and parents to understand the basic meaning without further interpretation. Once developed, the teacher's grade meaning statement should be communicated multiple times to students and parents throughout each school year. This begins at the start of the year when teachers explain the grade meaning to their students and provide them with a syllabus that includes the grade meaning statement. But in addition to reciting the statement, it is important for teachers to provide students with the rationale for this grade meaning. Students need to understand why grades require a change from traditional practices. In order to shift grading culture, students need to comprehend that grades are often misused for purposes other than communicating student learning. Teachers should then continue to remind students about the meaning of grades in the class at least once per quarter and provide an opportunity for class discussion on the topic. It is also important to communicate the meaning to parents - ideally in person - at least once per school year. This opportunity may arise in parent-teacher conferences, back-toschool night, or even in individual phone calls home. Face-to-face communication allows parents to better understand the meaning of the grade and have an opportunity to discuss any questions or concerns they may have. These conversations about grades with students and parents are key factors in transforming grading culture.

\section{Aligning Theory to Practice}

Beyond creating and communicating the grade meaning statement, meaningful grade creation requires teachers to apply grading theory to practice. To do so, teachers should ensure that all grades represent only student learning of the curriculum at the end of a learning unit. This requires a reflection process in which teachers consider the purpose of each of their

\section{Meaning of Grades: Grades appearing on assessments and report cards represent the student's learning of specific academic standards in the class. See the back of the page for the pacing of standards throughout each quarter.}

Figure 1. Example grade meaning statement appearing on a syllabus aligned to researcher recommendations.

assessments and assignments to ensure alignment to the established grade meaning. Through reflection, teachers ensure purposeful assessments and assignments that result in more valid student grades.

The grade reflection process may best begin with assessment review. Assessments that are used to measure student learning progress, often termed formative assessments or quizzes, should not receive grades because they are used in the middle of the learning process. They are meant to determine what additional learning, if any, a student requires for a particular standard, so assigning a grade representing summative learning would be unfair and inaccurate. Instead of grades on formative assessments, teachers should provide detailed feedback, rubric scores, or other marks that are not recorded in the gradebook. The other form of assessment, used to measure student learning at the end of a unit and often termed summative assessment, should receive grades. In fact, summative assessments should be the only instruments that 
teachers use to measure summative learning and therefore the only thing in a class receiving a grade. This reliance on summative assessments to create report card grades also means that teachers must do their best to ensure these assessments accurately assess student learning of the standards explicitly taught in class. While this does not require teachers to be psychometricians, it does require them to be purposeful in assessment creation.

Grade reflection is also useful to ensure purposeful assignments. Throughout a long school year, student assignments, in the form of classwork and homework, are especially prone to lack a meaningful purpose. Whether misused at times as "busy work" or to instill rigor in students through daily homework, assignments often stray from a optimal purpose of practicing academic standards and fostering learning that will later be assessed on summative assessments. Those assignments not aligned to this purpose should be revised or eliminated. As this assignment purpose is much different from the assessment purpose of measuring learning, assignments should not receive a grade. Because practice is used during the learning process, it would not be fair or accurate to assign students a grade that represents their learning on a standard when they are still in the middle of the learning process.

After ensuring assessment and assignment alignment to the grade meaning statement, the report card grade becomes clearer and simpler to create and interpret. Because the research-aligned grade represents only student learning of academic standards explicitly taught in the class, the final report card grade can only be composed of summative assessment grades. As the typical grading quarter lasts between eight and twelve weeks and a learning unit typically lasts a minimum of two weeks, it is likely that a report card grade representing an academic quarter would contain four to five assessments and hence four to five total grades. While recommending a particular method of combining these four to five assessment grades to create a report card grade is beyond the scope of this article, the teacher should be mindful that the final grade is truly representative of the student's learning of academic standards explicitly taught during the marking period.

\section{Anticipating Teacher Objections}

For the school leader looking to build support for reform across an entire department, school, or district, it is important to consider potential teacher objections --especially with an issue as contentious as grading. One potential objection is the need to define grade meaning all together. To educators with the understanding that grades have a universal meaning requiring no additional explanation, taking the time to explicitly define grade meaning may seem unnecessary. Although misguided, this concern does have some merit. It is true that the public's general understanding of report card grades is that they represent student learning (Brookhart, 2004). Unfortunately this general understanding is usually inaccurate, as the hodgepodge nature of grades often results in an unclear meaning consisting of both achievement and nonachievement factors (Randall \& Engelhard, 2009, 2010). But grading without a clearly established meaning is clearly poor practice. Further, decision makers who use teacher-created grades that lack an explicit meaning and are created in a hodgepodge manner are destined to make ill-informed decisions pertaining to student learning.

A second major objection will undoubtedly arise as some teachers question why students will complete assignments if they are not graded. Teachers with this concern believe students primarily complete assignments, quizzes, and tests because of the grades they receive, and without these grades, students have little to no motivation to complete any work. This type of concern represents a traditional and antiquated grading paradigm in which grades serve as a classroom currency and students are rewarded and punished for certain types of classroom behaviors (Guskey, 2015; Marzano, 2000). The necessary paradigm shift required to transform grading culture will take time, continued reflection, and dialogue within the school community to take hold and become internalized. But teachers with this objection need to understand that by grading assignments, the teacher often conflates effort and/or behavior with learning. Upon converting to a grade exclusively representing student learning and ensuring the purposeful alignment of assignments and assessments, teachers are often surprised to find that students continue to complete their work and take quizzes even without grades. In fact, upon shifting the grading culture to one centered on meaningful grades, each assignment and quiz can become more meaningful for students to truly practice their learning, gauge the breadth and depth of their learning of the standards, and prepare for the summative assessment--largely leaving the need to copy, cheat, and grade grub in the grading past.

\section{Conclusion}

Student grades that appear on assessments and report cards have no universal meaning and therefore require teachers to explicitly define and communicate the meaning in a clear manner to students and par- 
ents. Grading experts recommend the meaning to be student learning of academic standards, which requires teachers following this recommendation to create report card grades consisting only of summative assessment grades. In addition, grades strictly representing student learning require assessments and assignments that reflect and reinforce this meaning. However, establishing a sound grade meaning is not the end of the grading reform process--it is just the beginning. But this work does put educators on the path of grading reform, and their grading practices will be stronger as a result. Further, by doing so reformers begin the important, yet challenging process of transforming grading culture. With over 100 years of poor grading practices in our country, even the most zealous reformer cannot enact grading reform over night. But a focus on clearly establishing grade meaning can be the catalyst for this long-awaited reform that students so desperately deserve.

\section{References}

Brimi, H. M. (2011). Reliability of grading high school work in English. Practical Assessment, Research \& Evaluation, 16(17). Retrieved from http:// pareonline.net/getvn.asp? $\mathrm{v}=16 \& \mathrm{n}=17$

Brookhart, S. M. (1991). Grading practices and validity. Educational Measurement: Issues and Practice, 10(1), 35-36.

Brookhart, S. M. (1994). Teacher grading: Practice and theory. Applied Measurement in Education, 7(4), 279-301.

Brookhart, S. M., Guskey, T. R., Bowers, A. J., McMillan, J. H., Smith, J. K., Smith, L. F., \& . . Welsh, M. E. (2016). A century of grading research. Review of Educational Research, 86(4), 803-848.

Dueck, M. (2014). Grading smarter, not harder: Assessment strategies that motivate kids and help them learn. Alexandria, VA: ASCD.

Guskey, T. R. (2015). On your mark: Challenging the conventions of grading and reporting. Bloomington, IN: Solution Tree Press.

Kunnath, J. P. (2016). The impact of school poverty level on the teacher grading decision-making process (Doctoral dissertation). Retrieved from ProQuest Dissertations \& Theses Full Text: The Humanities and Social Sciences Collection. (Accession No. 1762747575)

Marzano, R. J. (2000). Transforming classroom grading. Alexandria, VA: ASCD.

Randall, J., \& Engelhard, G. (2009). Examining teacher grades using Rasch Measurement Theory. Journal of Educational Measurement, 46(1), 1-18.

Randall, J., \& Engelhard, G. (2010). Examining the grading practices of teachers. Teaching and Teacher Education, 26, 1372-1380.

Reeves, D. B. (2011). Elements of grading: A guide to effective practice. Bloomington, IN: Solution Tree Press. 